Archives

\title{
L'histoire scientifique et les utilisations antimodernistes du passé dans le médiévisme américain
}

\section{Gabrielle M. Spiegel}

Traducteur : Cécile Soudan

\section{OpenEdition Journals}

\section{Édition électronique}

URL : http://journals.openedition.org/ccrh/2422

DOl : $10.4000 /$ ccrh. 2422

ISSN : $1760-7906$

\section{Éditeur}

Centre de recherches historiques - EHESS

\section{Édition imprimée}

Date de publication : 20 avril 1999

ISSN : 0990-9141

\section{Référence électronique}

Gabrielle M. Spiegel, «L'histoire scientifique et les utilisations antimodernistes du passé dans le médiévisme américain », Les Cahiers du Centre de Recherches Historiques [En ligne], 22 | 1999, mis en ligne le 17 janvier 2009, consulté le 19 avril 2019. URL : http://journals.openedition.org/ccrh/2422 ; DOl : $10.4000 /$ ccrh.2422

Ce document a été généré automatiquement le 19 avril 2019

Article L.111-1 du Code de la propriété intellectuelle. 


\title{
L'histoire scientifique et les utilisations antimodernistes du passé dans le médiévisme américain
}

\author{
Gabrielle M. Spiegel \\ Traduction : Cécile Soudan
}

1 Le titre de ma série de conférences « Dans l'œil du miroir » vient à la fois du nom de la revue la plus éminente consacrée aux études médiévales en Amérique du Nord, le journal Speculum, édité par la Medieval Academy of America, et de l'emblème qui, jusqu'au début des années quatre-vingt, a traditionnellement orné sa couverture. Ce choix, E. K. Rand l'expliquait dans la présentation de la revue :

Speculum, ce miroir auquel nous trouvons correct de donner un nom latin, évoque les miroirs innombrables dans lesquels les gens du Moyen Age aimaient à se voir, eux et les autres - il s'agit de miroirs d'histoire, de doctrine et de mœurs, de miroirs de princes, d'amants et de fous. Nous ne comptons faire aucune folie consciente, mais nous reconnaissons la satire, l'humour et la joie comme faisant partie de notre objectif. L'art, la beauté et la poésie sont une part de notre héritage médiéval. Notre contribution à la connaissance de cette époque doit être, tout d'abord, savante, mais cette érudition doit être revêtue, autant que possible, d'une forme plaisante ${ }^{1}$.

Dans l'espoir de créer une excellente revue, pour Rand et les cofondateurs de l'Académie, les études médiévales, en Amérique, devaient surmonter les préjugés et la contamination idéologique entachant le terme «médiéval » qui, à travers les siècles, connotait un âge sombre, arriéré, superstitieux et "gothique», ce que Karl Marx appela un jour la «zoologie» de l'homme, son histoire animale. Au contraire, l'image que le miroir, speculum, du médiéviste américain devait montrer était un portrait attirant de la profession. Pour symboliser cet objectif, les fondateurs ornèrent la couverture de la revue d'une gravure où, devant le regard du lecteur-observateur, une main tenait un miroir vide, sans image. Icône du journal aussi bien que des études que les fondateurs espéraient promouvoir, ce miroir, dépourvu d'image, invitait donc le médiéviste à projeter sa propre image sur la surface vide. Il fallait un investissement volontaire du «moi», une 
implication narcissique, pour engendrer les images à travers lesquelles on pourrait contempler le sens du passé.

3 Dans le choix du nom et de la glose iconographique, les fondateurs offraient, sans en être conscients, un symbole efficace de la situation essentielle de l'histoire médiévale en Amérique: l'absence. Car, seule parmi les pays revendiquant un lien originel avec l'Europe occidentale, l'Amérique n'a pas de passé médiéval. Toute tentative pour affirmer l'importance et la pertinence de l'histoire médiévale doit donc d'abord surmonter (ou réprimer) son altérité absolue et son manque de lien, visible et partagé, avec tout passé national ou culturel « américain ».

4 L'« altérité » du Moyen Âge, bien sûr, n'est pas propre à la conscience américaine. Comme Lee Patterson le dit, le Moyen Âge a longtemps servi à la conscience historique occidentale postmédiévale de site principal d'altérité par rapport auquel elle pouvait se constituer ${ }^{2}$. Tel qu'il est construit par les humanistes de la Renaissance, le Moyen Âge comprend «l'autre» ténébreux de l'Occident, contre lequel la Renaissance et la modernité mêmes furent définies. Patterson résume :

L'humanisme, le nationalisme, la prolifération de systèmes de valeurs concurrents, la compréhension sûre d'une conscience historique, l'idée de l'individu, la production esthétique comme une fin en soi, la conception du monde naturel en tant que site de l'exploitation coloniale et d'investigation scientifique, la sécularisation de la politique et de l'idée d'État - toutes ces caractéristiques, et beaucoup d'autres encore, pense-t-on, mettent la Renaissance à part du Moyen Âge et, en même temps, l'alignent sur le monde moderne ${ }^{3}$.

Dans cette perspective, le Moyen Âge est précisément un millénaire de médiocrité, un espace d'attente vide et de véritable mort, jusqu'à la réanimation de l'Ouest en sa propre nature et en sa raison d'être, par la Renaissance.

5 Pour les Européens, le Moyen Âge, s'il n'est pas moderne, est au moins « là », évident dans les monuments qu'il a érigés et dans les traditions qui se trouvent, dit-on, à l'origine des États nationaux européens, modernes. C'est une des particularités des études médiévales, en tous lieux, que d'osciller entre deux saisies contradictoire du Moyen Âge : non-origine et période sombre, voire mortelle, ou origine de l'État moderne. Pris dans ce double sens de manque et de plénitude, le Moyen Âge, selon Kathleen Biddick, peut être

[...] partout, à la fois médiéval et moderne, et nulle part, sublime et rédempteur ${ }^{4}$.

C'est, en partie, cette altérité - ce fait d'être «autre » du Moyen Âge - qui donne aux médiévistes le sentiment d'une légitimité professionnelle. L'étrangeté et la différence, signifiées par l'éloignement, suggèrent qu'une qualité spécifique est nécessaire à l'étude de cette période. En Amérique, l'ambivalence est plus aiguë encore en raison de cette altérité absente : c'est pourquoi les premiers érudits américains insistèrent, exagérément, sur la pertinence du Moyen Âge, origine du monde moderne, et donc américain. Pour surmonter l'absence et l'étrangeté, les premiers médiévistes, en Amérique, interprétèrent l'altérité comme une identité. Cela dit, il n'est guère surprenant que l'étude de l'histoire médiévale aux États-Unis soit, depuis le début, marquée par des paradoxes internes qu'il est nécessaire de mettre au jour.

6 La civilisation médiévale évoque le passé triomphal du "catholicisme», de la «culture gothique » et d'un monde organisé selon la tradition et la coutume. En revanche, ses premiers historiens, aux États-Unis, furent plutôt des fondateurs protestants, éclairés et révolutionnaires ${ }^{5}$. Jefferson et d'autres révolutionnaires américains étaient plongés dans les mythes de la démocratie anglo-saxonne, dont les lois et les chroniques, pensaient-ils, préfiguraient leurs ambitions démocratiques. Jefferson se sentait si endetté envers la 
culture anglo-saxonne et ce qu'il prenait pour un régime de démocratie sociale, qu'il projetait de représenter deux héros anglo-saxons - Hengist et Horsa, invités en Bretagne par Vortigern, selon l'Historia Ecclesiastica de Bède, pour défendre le pays contre les ennemis du nord - sur le grand sceau de la nouvelle république. Son revers devait porter une image de la colonne de feu qui avait conduit le peuple élu vers la Terre promise (Exode, XIII, 21-22). Selon John Adams, à qui il communiqua ce désir, Jefferson voyait Hengist et Horsa comme représentant "[leur] forme de gouvernement " ${ }^{6}$. Il enracinait donc les institutions démocratiques américaines dans les pratiques sociales des peuples préchrétiens germaniques ${ }^{7}$. Jefferson n'avait pas lu Bède soigneusement. Ce dernier montre en effet que, bien que les guerriers anglo-saxons fussent arrivés sous l'apparence de protecteurs de l'Angleterre, « leur intention réelle était, néanmoins, de la réprimer ». Ensuite, Hengist devint le fondateur d'un lignage royal ${ }^{8}$. Pis encore, la colonne de feu de l'Ancien Testament signifiait, non la direction et la protection, mais la conquête, et illustrait les ambitions territoriales du jeune pays'.

7 Un autre projet fut soumis par un comité - composé de Benjamin Franklin, John Adams et Thomas Jefferson - chargé de préparer un emblème pour un sceau des États-Unis. Il était aussi paradoxal que celui dont il vient d'être question et fut également rejeté par le Congrès continental. Ce comité, s'inspirant d'un plan suggéré par Pierre-Eugène du Simitière, peintre suisse émigré qui vivait à Philadelphie, proposait que «le sceau des États-Unis d'Amérique ait à son centre un bouclier divisé en six sections». Les sections contiendraient, respectivement, les armes médiévales de l'Angleterre, de l'Écosse, de l'Irlande, de la France, de l'Allemagne, de la Belgique et des Pays Bas, «... pays qui peuplèrent ces États ». Le bouclier lui-même était tenu, d'un côté, par la "déesse de la Liberté en cuirasse », et, de l'autre, par la déesse de la Justice; au-dessus aurait flotté "l'Æiil de la Providence dans un triangle radieux ", et au-dessous aurait été inscrite la devise E Pluribus Unum. Par conséquent, comme l'indique Jay Fliegelman, le sens de cette devise, dans sa première occurrence officielle, faisait référence, autant au processus par lequel l'Amérique provient des six pays qui peuplèrent ses États, qu'à l'Amérique en tant qu'union d'États ${ }^{10}$. Cette représentation des États-Unis tourne le dos à l'interprétation révolutionnaire, rupture rebelle avec le passé. Le sceau figure l'Amérique comme une union européenne, et souligne la continuité du pays par rapport à ses origines européennes, auxquelles, contre la Déclaration d'indépendance, il refuse de renoncer. C'est sans doute une des raisons principales pour lesquelles la proposition du comité fut rejetée. Finalement, le Congrès continental - le drapeau fut choisi en juin 1777 - préféra la forme abstraite des treize bandes rouges et blanches alternées, signifiant « la vigueur et la vaillance ». Cette forme ne stipulait aucune origine, et mettait l'accent rhétorique sur la révolution comme rupture et indépendance, plutôt que comme continuité ${ }^{11}$. La dialectique entre l'indépendance et l'origine, la liberté d'action et la dépendance, et les contradictions que l'emploi de ces figurations médiévales imprima sur l'avenir américain demeurèrent un trait caractéristique de la quête de l'identité américaine et des origines, dans un passé absent.

Dorothy Ross a repéré très soigneusement la manière dont les penseurs américains, à l'époque postrévolutionnaire, fondent une vision "préhistoriciste» de l'exception américaine : le fondement révolutionnaire de la république, lié à l'existence d'un énorme réservoir de terres, doit préserver la vertu républicaine ainsi que le caractère agrarien du pays qui pourra, ainsi, avancer vers un avenir inchangé. Cet « investissement millénaire de la République américaine », comme Ross le montre, «fait du passé, le prologue, et de 
l'avenir, l'accomplissement d'une destinée républicaine ». Renforcé par le nationalisme et la religiosité protestante, l'exceptionalisme se révèle comme constituant, durant tout le $\mathrm{XIX}^{\mathrm{e}}$ siècle, un cadre durable de pensée historique ${ }^{12}$. La nature anhistorique (ou préhistoriciste) de l'exceptionalisme américain n'empêcha pas les historiens américains, qui travaillaient sous l'égide de cette providence particulière, de rapprocher leur récit national de celui de la liberté anglo-saxonne, développé en Angleterre ${ }^{13}$. Bien qu'ils aient abandonné l'idée d'enraciner, emblématiquement, les destinées américaines dans les passés nationaux européens, c'est-à-dire dans une continuité d'entités politiques, les Américains ne renoncèrent pas à l'idée d'une filiation. Mais ils optèrent pour des continuités raciales. Et c'est là, dans son legs "racial», que le Moyen Âge acquit sa prééminence dans l'imaginaire historique des écrivains et des historiens américains du $\mathrm{XIX}^{\mathrm{e}}$ siècle et qu'il contribua à la définition de la société américaine comme nécessairement blanche, anglo-saxonne et (ironiquement) protestante.

Le médiévisme populaire, aussi bien en Angleterre qu'en Amérique, surgit à l'époque romantique. Il s'inspire des études européennes sur le $\mathrm{xVIII}^{\mathrm{e}}$ siècle consacrées aux origines de la liberté occidentale, mise en œuvre dans leurs forêts, par les peuples teutoniques. Les manifestations et les applications du médiévisme teutonique sont si pénétrantes et si diverses que lord Acton considérait la « découverte d'un palimpseste [médiéval], ce renouvellement d'une continuité interrompue ", comme étant la "grande œuvre du XIX ${ }^{\mathrm{e}}$ siècle $»^{14}$. En Angleterre, de nombreuses branches du médiévisme sont liées au conservatisme anglican et au réveil d'une «haute ecclésiologie »; en Amérique, la résurrection médiévale est, en gros, le travail de protestants ou d'agnostiques ${ }^{15}$ qui s'attachèrent à l'étude des origines de la liberté anglo-saxonne, liant l'autogouvernement américain à une tradition continue remontant aux tribus teutonnes. D'inspiration et d'exécution whig, la thèse " teutoniste " prétendait que les institutions gouvernementales américaines étaient le dernier anneau d'une chaîne ininterrompue de pratiques tribales, primitives, germaniques, d'autogouvernement. Importées par les Anglo-Saxons en Angleterre, temporairement submergées par le «joug normand», puis réapparues intactes comme principes constituants de la Magna Carta et de la Révolution glorieuse, elles auraient été apportées, de l'autre côté de l'océan, par les colonisateurs, puis perfectionnées sur les rives de la Nouvelle-Angleterre, dans la Révolution et la Constitution américaines ${ }^{16}$. La liberté s'enracine donc dans l'appartenance de race; elle est transmise par la descendance généalogique du peuple, indépendamment du lieu dans lequel elle peut prendre racine et fleurir.

Dès 1840, les Américains pouvaient suivre des conférences publiques, présentées par des hommes comme Asahel Davis, sur la prétendue colonisation viking de la NouvelleAngleterre. Ce point de vue fut solidement étayé, deux décennies plus tard, par Eben Horsford, connu pour son talent de vulgarisateur, qui chercha à rattacher des noms du norvégien ancien à différents sites autour de Boston ${ }^{17}$; dans les années 1860 et 1870 se répandirent les rêveries inventives de John Fiske sur le folkmoot, assemblée du peuple anglo-saxon, et germe de la liberté démocratique ${ }^{18}$. En Amérique, les grands historiens romantiques $\mathrm{du}$ milieu $\mathrm{du} \mathrm{xIX}^{\mathrm{e}}$ siècle-Bancroft, Prescott, Motley et Parkman - partageaient l'idée que le gène libertaire essentiel qui formait les traditions démocratiques était d'origine teutonique, et tous opposaient les races teutonnes, friandes de liberté, aux peuples celtes et " méridionaux $»^{19}$. Prescott, écrivant l'histoire espagnole, soulignait l'origine wisigothique des Castillans. Il attribuait à cette origine, la précoce apparition de traditions castillanes de liberté constitutionnelle, tout en signalant que de 
tels germes passent mieux " par d'autres nations et sous de plus heureux auspices ", où, par contraste avec le régime simple des Wisigoths, ils deviennent la base d'une « liberté constitutionnelle bien réglée $»^{20}$. Pour ces historiens, l'Allemagne est, comme l'indique Motley, dans son essai Historic Progress and American Democracy, « la mère commune des nations et des empires - alma mater felix prole; elle gouverne toujours la pensée de sa vaste couvée: les Francs, les Goths, les Saxons, les Lombards, les Normands, les Néerlandais, les Américains - tous allemands!! $»^{21}$. Il n'est pas surprenant que E. R. Curtius ait regardé l'intérêt des Américains pour le Moyen Âge comme une manifestation d'un "sens spirituel profond », qu'il compare au "sentiment de désir que nous penserions trouver chez l'homme qui se met à la recherche de sa mère perdue $\star^{22}$. Tout assurait aux traditions démocratiques une généalogie commode bien que spécieuse. La thèse teutonique projetait l'Amérique au sein de l'unité des races germaniques et démontrait une continuité, selon Motley, Bancroft et les autres, essentielle à la compréhension de l'histoire.

11 Les praticiens américains d'histoire médiévale, pendant cette période, étaient, pour la plupart, issus de familles distinguées de Nouvelle-Angleterre. Ils étaient attirés par l'époque médiévale, source-pensaient-ils - de leurs propres institutions et modèle romantique d'un monde qui - espéraient-ils - continuerait à reconnaitre leur position centrale en son sein. Repoussée vers les marges du monde politique américain durant l'époque qui précéda la guerre de Sécession, cette élite du Nord-Est, « après la guerre, se mit en devoir d'imposer son autorité et sa vision sociale du pays $»^{23}$. Elle était consciente que la composition sociale changeante des États-Unis résultait de la croissance de l'immigration, en particulier, après la guerre de Sécession, de l'arrivée massive de catholiques qui menaçait de submerger le caractère protestant de la société américaine. Elle était attentive à l'industrialisation qui tendait à créer de nouveaux conflits de classes. Elle se tenait prête à faire face au défi que la pensée scientifique du XIX ${ }^{e}$ siècle lançait aux croyances protestantes traditionnelles ${ }^{24}$. Ces patriciens de Nouvelle-Angleterre cherchaient à fonder leur autorité culturelle sur un mélange complexe de traditions culturelles et de modernité scientifique. Comme Robin Fleming le montre, ces spécialistes du passé médiéval, bien que profondément sceptiques quant aux possibilités du modernisme, étaient parallèlement branchés sur les derniers développements scientifiques. Leur médiévisme s'exprimait dans un langage mêlant l'idiome mécaniste de l'âge industriel, celui des nouveautés scientifiques (biologie, dont le darwinisme, géologie et philologie), avec «la terminologie occulte du protestantisme du XIX siècle. Il s'agit d'une langue - note Fleming - [remplie d'] impulsions paradoxales incitant à restaurer un monde perdu et à tout soigner par le progrès $»^{25}$. Du reste, conclut-elle :

Aux États-Unis, la volonté des médiévistes de faire revivre un monde perdu et de se doter d'un lignage ainsi que l'accueil fait à une science à moitié comprise se combinaient dans l'écriture fondamentalement idéologique du xixe siècle sur le Moyen Âge: la race est la force motrice de l'histoire et la «race aryenne », en particulier sa branche « teutonique », est supérieure à toutes les autres ${ }^{26}$.

Durant les dernières décennies $\mathrm{du} \mathrm{XIX}^{\mathrm{e}}$ siècle, ce qui, chez les historiens romantiques, avait fonctionné comme simple motif et comme explication générale (nonintégrée à leurs récits politiques) prenait progressivement un caractère « scientifique » dur, transformant la thèse teutoniste en une théorie "scientifique ", intégralement développée à partir du "germe teutonique ». Henry Adams, chez qui le médiévisme et l'enthousiasme pour la science se combinaient fort bien, est une parfaite illustration des tendances incompatibles et des inquiétudes culturelles que cache cette évolution. 
Henry Adams fut la première personne, en Amérique, à enseigner l'histoire médiévale, en tant que professionnel. Il raconta plus tard cette brève période de son début de carrière dans The Education, sous le titre « Failure ». Après avoir reçu son diplôme universitaire, à Harvard, Adams passa deux ans, sans guère de but, en Allemagne, à étudier le droit civil ; il apprit à lire les documents, utilisant la nouvelle méthode philologique allemande. Rejoignant son père, Charles Francis Adams, à Londres, il fut son secrétaire personnel. À l'époque de la guerre de Sécession, Adams s'enthousiasma pour Darwin, qui entraînait l'élite cosmopolite. Il rédigea un de ses premiers textes pour la North American Review : un exposé des Principles of Geology de Sir Charles Lyell. Ayant peu de devoirs officiels, il passait son temps à lire goulûment Auguste Compte, Alexis de Tocqueville et John Stuart Mill, «grands prêtres» de sa foi dans le libéralisme aristocratique et réformateur ${ }^{27}$. Au même moment, sur le chemin de "l'histoire scientifique ", il commença à étudier les œuvres de Henry Buckle et de Herbert Spencer, chemin qu'il poursuivit, souvent vainement et avec beaucoup de frustration, jusqu'à la fin de sa vie. Le voyage commença dans l'enthousiasme. Dans sa lettre sur "The Tendency of History" à l'American Historical Association, discours présidentiel de 1894, publié seulement après sa mort par son frère Brooks en 1919, Adams regarde vers ses premières années de lecteur, à Londres, et tente de communiquer l'émotion qu'il ressent à propos de ce qui est alors devenu une approche démodée du passé :

Personne, parmi ceux qui ont observé le cours de l'histoire durant la dernière génération, ne peut avoir de doutes sur son orientation. Ceux d'entre nous qui ont lu le premier volume de l'History of Civilization in England de Buckle, lorsqu'il a paru, pour la première fois, en 1857, et qui ont lu, presque immédiatement après, en 1859, De l'origine des espèces - et, qui ont ressenti l'envie véhémente qu'a donnée Darwin d'étudier les lois naturelles - n'ont jamais douté que les historiens suivraient ces préceptes jusqu'à ce qu'ils aient épuisé toute hypothèse possible en vue de créer une science de l'histoire ${ }^{28}$.

Les principes de l'évolution biologique, esquissés par Darwin, se combinaient au positivisme de Comte, surtout dans la version progressiste des positivistes anglais, Buckle et Spencer, pour offrir à l'historien une histoire véritablement "scientifique ». Pour Henry Adams, cette histoire scientifique ne se définit pas principalement par l'interprétation critique des documents et par l'établissement des faits, mais par une capacité à unifier le passé en une suite nécessaire et complète de mouvements laquelle - écrit Adams dans son compte rendu, pour la North American Review, de l'ouvrage de Van Holt, Constitutional and Political History of the United States - - ressemble, dans son mode d'opération, à l'action mécanique de la Nature même $»^{29}$. Ou, comme l'explique de manière plus complète Adams à Parkman :

Plus j'écris, plus je suis certain qu'avant longtemps une nouvelle école historique s'élèvera, qui nous dépassera... Je suis heureux que le développement purement mécanique de l'esprit humain [...] puisse apparaitre si clairement, dans une grande démocratie, grâce à l'absence d'obstacles [...; ] dans une génération à venir, la psychologie, la physiologie et l'histoire s'uniront pour prouver que l'homme a un développement aussi fixé et nécessaire que celui d'un arbre; et presque aussi inconscient ${ }^{30}$

Ici, Adams dévoile la dérive déterministe du raisonnement scientifique de la fin de l'ère victorienne qui, bien que spécialement marquée dans ses écrits, caractérise aussi bien les pensées européennes qu'américaines. Le "darwinisme » ${ }^{31}$ et le positivisme de Comte, pensait-on, pouvaient se combiner et faire entrer toute la vie humaine dans le réseau des lois déterministes mises au jour par l'enquête scientifique. Le comportement humain, 
passé et présent, considéré comme le révélateur les lois fondamentales qui régissent la vie sociale, pourrait dévoiler les structures de l'évolution, lesquelles organisent le chaos apparent de la vie humaine sur terre aussi sûrement qu'elles règlent les transformations morphologiques du squelette des vertébrés. La multiplicité pouvait donc se réduire à l'unité, un seul fil reliant le passé, le présent et le futur, dans un processus inexorable. À la fin du XIXe siècle, ces lois progressistes naturelles conduisaient vers des formes de vie plus élevées et meilleures.

Cet optimisme évolutionniste devint, aux États-Unis, une composante clé de l'histoire scientifique. Tandis que Henry Adams perdait son optimisme, d'autres le gardaient. Dorothy Ross montra que l'essor des sciences sociales et de l'historicisme au XIX ${ }^{\mathrm{e}}$ siècle a une fonction compensatoire, dans un monde social de plus en plus conscient de la nouveauté, de la contingence et de l'incertitude des affaires humaines. Cela valait surtout pour les élites qui constataient l'affaiblissement de leur autorité culturelle et de leur position sociale, dans un monde capitaliste en voie d'industrialisation rapide ; les sciences sociales «fournissaient des modèles pour montrer que cette inquiétude pouvait être soulagée par les effets stabilisateurs de la science et du progrès historique ».

En 1871, lorsque Charles W. Eliot, président de Harvard, invita le jeune Adams à enseigner l'histoire médiévale à l'université et à éditer la North American Review, Henry était marqué par cette science du milieu du siècle, découverte pendant son séjour à Londres. Toutefois, avec un ton lourdement ironique (L'Education) et tout en adressant des excuses à Henry Maine, Tyler, McLennan, Buckle, Comte ainsi qu'à différents philosophes, Adams affirme son absolue inadéquation aux études médiévales :

[...] l'homme, qui allait résoudre l'énigme du Moyen Âge et la situer dans le cours du passé au présent, serait un plus grand homme que Lamarck ou Linné; mais l'histoire n'avait nulle part échoué aussi pitoyablement; elle n'avait jamais déclaré aussi triste faillite. Depuis Gibbon, le spectacle en était presque scandaleux. L'histoire avait même perdu toute notion de honte. Elle avait cent ans de retard sur les sciences expérimentales; elle était moins instructive que Walter Scott et Alexandre Dumas ${ }^{32}$.

Adams confesse, au président Eliot, ne connaître aucun autre médiéviste américain, trahissant ainsi son ignorance de Henry Charles Lea, qui avait déjà produit trois œuvres sur la religion médiévale et qui s'était lancé dans son travail majeur sur l'Inquisition ${ }^{33}$. Lee était éditeur plutôt que professeur. En vérité, en histoire médiévale, l'Amérique n'avait aucun savant d'importance, qui soit comparable à ceux de l'Angleterre, de la France ou de l'Allemagne : aucune tradition sur laquelle Adams puisse se fonder, même pour la plus simple des tâches d'enseignement. Pour Adams, l'histoire comptait pour moins que rien si elle n'était pas scientifique. Il s'appuya donc sur la théorie « scientifique » principale de l'époque relativement à la période médiévale - la théorie du germe teutonique - qu'il incorpora dans le seul écrit qui sortira de son enseignement, "The Anglo-Saxon Courts Law », partie de l'œuvre globale publiée en 1876 sous le titre de Essays in Anglo-Saxon Law.

Nous sommes bien renseignés sur l'enseignement de Henry Adams à Harvard. Nous avons sa propre description, ironique, dans The Education, le témoignage du travail fait en séminaire dans les Essays, également un compte rendu de Herbert Baxter et le cahier d'un ancien étudiant, Charles Thwing, découvert à Western Reserve University (dont Thwing devint plus tard, président). Selon toutes ces informations, on peut créditer Henry Adams 
d'avoir introduit le séminaire de style allemand, dans la pédagogie médiévale des universités américaines.

Selon Herbert Baxter Adams, Henry Adams lança son séminaire dans l'idée d'introduire en Amérique l'érudition allemande de von Maurer et de Waitz, telle qu'elle pouvait être transmise par Sir Henry Maine et William Stubbs ${ }^{34}$. Selon tous les témoignages, Maine constitua un objet d'analyse pour Adams tant dans ses séminaires de premier et de deuxième cycle que dans ses séminaires doctoraux. Comme le rapporte Herbert Baxter Adams :

Le professeur Henry Adams a commencé son travail de séminaire par un examen critique des écrits de Sir Henry Maine. Ses élèves prenaient chacun un chapitre et l'étudiaient, sous divers éclairages. Chacun rendait compte à la classe des résultats de son étude critique; était repris sèchement, sur tout point douteux, par le professeur, dont les véritables points de vue sur le sujet n'étaient jamais avoués avant la clôture de l'exercice ${ }^{35}$.

De la même façon, le cahier de Thwing, couvrant les conférences de premier et deuxième cycle sur les institutions médiévales, indique que les textes de base du cours étaient l' Ancient Law et le Village Communities in the East and West de Sir Henry Maine ainsi que le Primitive Marriage et le Lex Salica de McLennan. Mais, comme cela est clair dans les notes, Adams n'avait recours à McLennan que de temps en temps, tandis qu'il traitait, chapitre par chapitre, l'œuvre de Maine, principalement l'Ancient Law ${ }^{36}$.

L'importance donnée à Maine apparaît également dans les comptes rendus que Adams fait de la recherche allemande et anglaise dans la North American Review. Article après article, Adams loue l'érudition allemande sur le Moyen Âge et exprime son mépris pour les Anglais, mépris auquel Maine, seul, échappe. Adams prétendait que c'était «le malheur de l'Angleterre de n'avoir encore jamais eu d'école historique scientifique $\aleph^{37}$. Il trouvait Stubbs insupportablement "ennuyeux», bien qu'il l'ait, en histoire institutionnelle, crédité de la compréhension de la « théorie du germe ». Il considère avec amusement l'adoption débridée, par Freeman, de ladite théorie. Il suggère que :

[...] incontestablement, l'objet patriotique qu[e Freeman] cible [c'est-à-dire une démonstration des origines teutoniques des institutions anglaises] serait atteint de façon beaucoup plus efficace s'il acceptait de légèrement refréner la vivacité réellement déchaînée de son enthousiasme, et de modérer un peu son allure, simplement pour ne pas disparaître de la vue de ses disciples essoufflés ${ }^{38}$.

Adams, bien que sa propre recherche médiéviste en soit aussi marquée et qu'elle soit enveloppée par un langage plus judicieux, se moque du déploiement extravagant de la théorie du germe teutonique dans le travail de Freeman. Cependant, rien ne démontre plus clairement son appropriation globale de cette théorie que son cours sur « The AngloSaxon Courts of Law ".

À cette époque, en Amérique, la "théorie du germe ", dans sa version "scientifique " intégrale, est relativement nouvelle. Elle porte en elle la généalogie historique prestigieuse de l'historiographie allemande et anglaise. Comme le dit Levenson, il s'agit

[...] d'une synthèse de nombreuses branches de l'érudition du xix siècle: la recherche des frères Grimm en philologie comparée, les études des institutions primitives (comme celles de Maine), la théorie évolutionniste de Herbert Spencer, et le racisme qui est le corollaire de toutes ces disciplines ${ }^{39}$.

La théorie du germe est décisive pour Adams ; elle lui permettait d'argumenter en faveur de la continuité essentielle de l'histoire, sans laquelle son étude ne pourrait jamais devenir scientifique, dans le sens positiviste de Comte. Ainsi, il avance, dans l'ouverture de «The Anglo-Saxon Courts of Law », que : 
Les travaux, longs et patients, des érudits allemands, semblent avoir maintenant établi, au-delà de toute contestation, le principe historique fondamental, à savoir que la famille germanique tout entière, dans sa phase de développement la plus tôt connue, a placé l'administration de la loi, comme elle a placé l'administration politique, dans les mains de l'assemblée populaire composée des membres libres et solides de la république. Ce principe [...] donne à l'histoire des institutions germaniques, et surtout anglaises, une complétude et une continuité philosophique, ce qui ajoute énormément à leur intérêt et même à leur valeur pratique.

L'historien, qui tente maintenant de retracer, à travers deux mille ans de vicissitudes et de dangers, le fil fragile de la pensée politique et juridique, ne le perd plus de vue dans la confusion de la féodalité, ou dans le désordre sauvage de l'heptarchie. Il le suit, sans incident et sans défaut, jusqu'à ce qu'il le mène, au moins, à l'ensemble primitif, composé de l'assemblée populaire, du parlement, de la cour judiciaire et de l'armée ; qui incluait tout homme libre, riche ou pauvre, et, en théorie, garantissait au moins des droits égaux à tous. Il se peut que l'origine de l'État et de la Loi soit ici ${ }^{40}$.

Adams s'efforce de démontrer, non seulement que l'existence d'une cour des "cent » (hundred), chez les Anglo-Saxons, fonde des développements judiciaires et constitutionnels tardifs, mais que l'organisation légale anglo-saxonne a anticipé et, dans la logique de la "théorie des germes », a engendré l'idée moderne d'État démocratique. Il insiste sur le fait que l'assemblée populaire allemande compose un district judiciaire et, chose importante, "géographiquement fixé ", fonctionnant comme la base du système administratif germanique ${ }^{41}$. Adams s'oppose ici à l'idée proposée par Maine, d'assemblées tribales primitives et les remplace par des institutions politiques authentiques. Donc, constate-t-il, la cour des «cent» est "parmi les premières, sinon la première des créations politiques de l'homme ». D'un bout à l'autre de son essai, Adams évite les termes tribu, gau et canton en faveur d'État, pour saisir la nature authentiquement politique des institutions en question ${ }^{42}$.

Rattachant la cour des " cent » aux districts territoriaux fixés et les considérant comme de véritables unités politiques et administratives, Adams pouvait soutenir que, pendant le règne d'Alfred, les institutions primitives importées en Angleterre par les Anglo-Saxons avaient atteint le statut de véritable système fédéral d'États. La descente dans l'anarchie de la féodalité et de la propriété privée qui se faisait déjà sentir en Europe continentale, à cause des réformes de Charlemagne, est ainsi retardée de plusieurs siècles. Il en était ainsi, parce que,

[...] parmi les races germaniques, aucune ne tient à ses coutumes et libertés anciennes, avec une indépendance plus constante ou avec un conservatisme plus tenace, que la grande Confédération saxonne, qui, si souvent et si profondément, imprime son caractère sur l'histoire de l'Europe du Nord ${ }^{43}$.

La «particularité distincte » de l'Angleterre, lorsqu'elle est comparée à l'Allemagne - une distinction « riche en intérêt philosophique », note Adams - est que

[...] le développement de l'Allemagne était sur le chemin d'une consolidation politique ; celui de l'Angleterre était sur le chemin de la confédération politique ${ }^{44}$.

Les "germes » teutoniques, en raison de la nature conservatrice des Anglo-Saxons, ont réussi à créer une fédération primitive qui est, selon Adams, l'ancêtre direct du système gouvernemental américain que

[...] ses deux propres ancêtres présidentiels ont préconisé et mis en place ${ }^{45}$.

Son succès en Angleterre est dû à la persistance de traits raciaux déterminés qui définiront plus tard le monde démocratique américain. Il est évident que "l'heureux exceptionnalisme » anglais - analogue, peut-être, à celui de l'histoire américaine elle- 
même ${ }^{46}$ - se termine avec Édouard le Confesseur, «normand, par naissance ; totalement normand par son éducation et ses sympathies $\aleph^{47}$. Le pays dut alors se soumettre à « l'aristocratie et à la féodalité » qui assaillit le continent. Jusque là,

[...] l'Angleterre se maintint saine et sauve à travers les périodes les plus sombres de

l'histoire moderne, au moment où tout espoir de bonheur semblait éteint dans le

nord de la France ${ }^{48}$.

L'explication est raciale et le but, comme pour toute pensée historique de la continuité, est de créer une identité ; il s'agit de construire une identité américaine à l'intérieur d'un modèle historique fourni par un passé racial et politique inventé.

Henry Adams, premier professeur et historien professionnel de l'époque médiévale, à Harvard (de manière brève), est une figure ambiguë et transitionnelle de l'histoire du médiévisme américain. Il se tint en partie à l'écart de la profession en tant que telle. Il demeura davantage un gentilhomme savant qu'un véritable professionnel. Intellectuellement, ses sympathies instinctives le conduisirent assez tôt vers les synthèses scientifiques potentiellement portées par le positivisme. Mais, comme John Higham ${ }^{49}$ et d'autres l'ont noté, l'histoire professionnelle, en Amérique, s'est développée en opposition au positivisme ; elle est passée par l'adoption d'un historicisme à la Ranke. Le désenchantement d'Adams par rapport à l'histoire médiévale fut assez rapide; avant même son départ de Harvard en 1877, il confiait déjà la plupart de son enseignement à son étudiant, Cabot Lodge, se tournant vers des sujets américains. Quand Adams revint à l'histoire médiévale dans Mont Saint-Michel and Chartres (1904), ce fut sur une base différente et avec des soucis distincts, non moins «scientifiques » que l'histoire juridique et institutionnelle qu'il pratiquait auparavant. Ce n'est donc pas à Henry Adams (à Harvard), mais plutôt à Herbert Baxter Adams (à Johns Hopkins) qu'il faut attribuer l'introduction, en Amérique, de l'histoire « scientifique » sur le modèle allemand.

\section{L'école scientifique}

L'essor de l'école « scientifique » historique américaine, dans les années 1870 et 1880, est, en grande partie, le résultat des espoirs mis dans le modèle allemand, au cours des décennies qui comptèrent dans la formation d'une histoire médiévale professionnelle. Ce n'est pas ici le lieu de répéter l'évolution de la professionnalisation de l'histoire américaine, déjà traitée par John Higham, Dorothy Ross, Peter Novick, W. Stull Holt et Jurgen Herbst, entre autres. Mais le caractère spécifique de l'érudition américaine, en histoire médiévale, ne peut être compris sans un aperçu, d'une part, sur les principes de l'école "scientifique " et, d'autre part, sur la place et les usages du médiévisme à l'intérieur de celle-ci. On se concentra sur le travail de Herbert Baxter Adams à l'université Johns Hopkins pour illustrer le processus de développement à un niveau plus général : en effet, il représente ce mouvement au sens large et parce que les excès mêmes de l'histoire « scientifique » à Hopkins dévoilent son cheminement, dissimulé ou non.

Lorsque Herbert Baxter Adams décide, en 1874, d'aller en Allemagne pour acquérir une formation approfondie en histoire, il suit un chemin que les Américains empruntaient depuis le début du siècle. Environ neuf mille Américains avaient été inscrits, avant 1900, dans des universités allemandes, dans divers diplômes supérieurs ${ }^{50}$. Le choix de l'Allemagne, plutôt que de l'Angleterre ou de la France, peut s'expliquer par l'absence d'enseignement vraiment professionnel en Angleterre - la science et la philosophie fleurissent en dehors des universités -, tandis que la France était tachée par «l'incroyance» propre aux Lumières, toujours suspecte aux yeux de l'Amérique protestante. Les parents américains, rapporte Herbert Baxter Adams, considéraient Paris 
comme « un endroit dangereux pour un jeune homme ${ }^{51}$. Pour développer une éducation supérieure, l'Allemagne l'emporte sur les autres pays. Les Américains, intéressés par les nouvelles méthodologies qui y étaient proposées, vinrent en grand nombre à Halle, Göttingen, Berlin, Leipzig et Heidelberg où ils étudièrent la philosophie, la théologie, la médecine, l'histoire et l'économie politique. De plus, l'accès aux universités allemandes était facile (l'inscription n'étant qu'une formalité). Les exigences, pour les diplômes minimaux, consistaient, en général, à deux ans d'assistance aux cours; pour obtenir le doctorat, une thèse acceptable et la réussite d'un examen général suffisait ${ }^{52}$. Ce que les Américains cherchaient principalement en Allemagne, c'était le prestige et la formation technique conférés par la Wissenschaft allemande. Dans l'érudition historique, les deux sont indissolublement liés au nom de Ranke, qui fonda l'investigation historique, utilisant les méthodes de la philologie critique, élaborées auparavant à Göttingen, et donnant à sa pratique un encadrement institutionnel par la création du premier séminaire, vers 1830 . La « méthode » scientifique de Ranke ainsi que le système du séminaire devaient devenir les clefs de voûte des innovations pédagogiques d'Adams à Hopkins, après son retour en Amérique.

On parle beaucoup du «malentendu » américain à propos de Ranke ainsi que de l'usage, particulièrement réductionniste, pour ne pas dire grossier, de sa méthode. Selon George Iggers, les Américains ont uniquement saisi l'accent que Ranke plaçait sur l'analyse critique des sources, Quellenkritick, en vue d'établir objectivement les faits. Les Américains identifiaient la pratique historique «scientifique »-abrégée en méthode « rankéenne »-avec un empiricisme strict qui avait tendance à s'enfoncer dans le factualisme: le but étant d'établir wie es eigentlich gewesen-peu de tentatives de généralisation et un rejet rigoureux de toute philosophie spéculative. Cette façon de concevoir Ranke, selon Iggers, est passée complètement à côté des présupposés métaphysiques de sa pensée - idéalisme romantique, universalisme et Identitätsphilosophie allemande -, selon lesquels l'étude de l'histoire était une forme de participation à une réalité divine, inhérente à l'histoire même ${ }^{53}$. Le Ranke "allemand», en contraste avec la version américaine, était un homme profondément immergé dans les causes philosophiques et religieuses. Il voyait, dans toute l'histoire, le fonctionnement d'idées et d'énergies morales d'origine divine, ce qui explique sa célèbre remarque selon laquelle « tous les âges sont également proches de Dieu ».

Ces déviations $\mathrm{du}$ "père de la science historique » sont peut-être exagérées. Il est vrai, cependant, que les Américains en général, et Herbert Baxter Adams en particulier, avaient tendance à invoquer Ranke comme garant d'une notion totalement " objective " et "scientifique» de l'enquête historique. Baxter Adams, dans un article de 1888 sur "Leopold von Ranke », oppose le penchant moral et philosophique qu'il discerne dans la lecture, par Niebuhr, de l'histoire romaine, aux pratiques de Ranke. Il loue ce dernier pour être [un historien]

[...] déterminé à se tenir strictement au fait, à ne prêcher aucun sermon, à n'indiquer aucune morale, à ne conter aucune fable, mais à dire la simple vérité historique. Sa seule ambition était de narrer les choses telles qu'elles étaient vraiment: wie es eigentlich gewesen ist. La vérité et l'objectivité étaient les buts les plus élevés de Ranke. L'histoire ne vise pas le divertissement ni l'édification, mais l'instruction... Il ne croyait pas être du ressort de l'historien d'indiquer la providence divine dans l'histoire humaine ${ }^{54}$. 
Par contre, dans «Is History Past Politics?», Adams loue Niebuhr comme étant un des fondateurs de ce qu'il appelait l'école « historico-politique » et reconnaît l'universalisme de Ranke.

Ranke croit en l'unité et en l'universalité de l'histoire... L'histoire est, dans sa nature même, universelle, dit Ranke... Il traite les pays individuels, l'Allemagne, la France et l'Angleterre non pas comme des nations isolées, mais comme des illustrations d'idées macro-historiques sur la religion, sur la liberté, sur la loi et sur le gouvernement, exprimées ou réalisées par différents États européens. Pour Ranke, comme pour Abélard, le grand clerc du Moyen Age, l'universel peut être distingué dans le particulier. Même l'histoire locale peut être traitée comme une partie de l'histoire générale ${ }^{55}$.

La référence que fait Adams à l'école « historico-politique » est significative ; elle reflète la formation qu'il a reçue en Allemagne, dans les séminaires de Bluntschli, Erdmannsdörffer, Droysen et Treitschke, les hommes qui ont inspiré le plus directement les premières conceptualisations historiques d'Adams ${ }^{56}$.

Comme toutes les branches de la science sociale allemande du $\mathrm{XIX}^{\mathrm{e}}$ siècle, les enseignements de l'école «historico-politique»-de Bluntschli, Erdmannsdörffer et autres - comportent une forte orientation historiciste, qui propose que toute forme de vie sociale soit étudiée en prenant en considération le développement cumulatif et le contexte spatio-temporel. Fondamentalement organiciste, l'historicisme allemand met l'accent sur la continuité de vie des sociétés, qui peut être retracée, surtout dans ses institutions politiques, c'est-à-dire dans l'État même. Comme l'a démontré Herbst, l'étude comparative d'institutions en voie de développement, en Allemagne et par conséquent en Amérique, "tire ses principaux outils conceptuels de la science biologique ${ }^{57}$. Théorie darwinienne de l'évolution, d'un côté, et large lexique de métaphores et d'analogies biologiques, de l'autre : c'est donc la biologie et la théorie de l'évolution qui fournissent la deuxième voie par laquelle l'histoire, à la fin du XIX ${ }^{\mathrm{e}}$ siècle, peut devenir "scientifique ".

L'utilité des métaphores biologiques et évolutionnistes pour l'analyse historique se trouve dans le concept de "changement cumulatif et constant, fonctionnant dans une chaîne infinie de causes et d'effets tangibles $\aleph^{58}$. Cette conception d'une causalité et d'une continuité génétiques est fondamentalement compatible avec la perspective historiciste. Cet historicisme génétique, pour Higham, correspondait aux intérêts pratiques de l'élite culturelle de la fin du XIX ${ }^{\mathrm{e}}$ siècle, attachée à restaurer son autorité culturelle sur la vie intellectuelle américaine. Appliquée à l'histoire institutionnelle, la leçon de la biologie évolutionniste comme développement continu et ininterrompu, enseigne également « un respect sobre de la dépendance du présent par rapport au passé $»^{59} \mathrm{et}$, donc, soutient les objectifs conservateurs de la coopération. En même temps, elle émancipe les nouveaux historiens «scientifiques » du souci romantique de l'individualité et de la personnalité. Elle place l'histoire dans un cadre externe et impersonnel d'institutions et d'États, renforçant, semble-t-il, son statut authentiquement « objectif ».

La vision évolutionniste des institutions politiques et sociales, enseignée en Allemagne, portait en elle une série d'hypothèses qu'Adams adopta et qui devinrent la pièce centrale de son travail de séminaire, à Hopkins. Chez Bluntschli - qui affirmait que « l'État en tant que personne historique est la création et l'expression de la communauté raciale de la nation »-, elles prenaient la forme de doctrines raciales sur la supériorité des peuples aryens $^{60}$. Bien qu'Adams n'épouse jamais l'aryanisme de son mentor, il étudia néanmoins les « germes teutoniques » des institutions américaines, et ce fut le noyau substantiel et le plan conceptuel de son séminaire de Johns Hopkins sur l'histoire et la politique. Ce 
faisant, il soutenait implicitement que ce qui définissait les institutions américaines dans le présent, c'étaient leurs origines médiévales ou protomédiévales. Pourquoi, aux ÉtatsUnis, l'histoire médiévale et l'histoire américaine sont-elles les deux sujets les plus étudiés par la profession naissante? La réponse est que en profondeur l'histoire médiévale rend compte, le plus nettement possible, de l'histoire américaine ; ce qu'elle a été et ce qu'elle doit continuer à être: le développement fructueux de ces "germes teutoniques » de liberté et de démocratie. Comme les institutions municipales qu'Adams fait remonter aux origines teutoniques, ces germes

[...] ont été répandus en Nouvelle-Angleterre par d'anciens vecteurs, anglais et allemands: importés par des pèlerins et des puritains et prêts à prendre racine, dans la terre libre de l'Amérique, comme le serait un grain égyptien qui sèche depuis des milliers d'années dans un sarcophage de momie ${ }^{61}$.

Tout lecteur des travaux du séminaire de Johns Hopkins, sur l'histoire et la politique, acceptant avec un enthousiaste noncritique la théorie du "germe teutonique ", est tenté de rejeter ce que Gettleman a appelé le "racisme rhapsodique ${ }^{62}$ pour son absurdité ingénue. Vue, aussi bien à partir du travail de $\mathrm{E}$. A. Freeman - ami proche et protecteur de Baxter Adams, dont la formule "L'histoire est la politique au passé et la politique l'histoire au présent » décorait la salle de séminaire - que de l'historiographie allemande, la théorie du germe offrait au nouveau séminaire d'histoire et de politique, selon Gettleman,

[...] une synthèse intellectuelle créative et d'une grande portée [...] liant diverses branches intellectuelles et politiques: l'expansion impériale, le racisme, la philanthropie scientifique, l'économie réformatrice et une variété d'initiatives de recherche, en histoire sociale américaine ${ }^{63}$.

Baxter Adams voyait les origines municipales de Nouvelle-Angleterre dans les germes teutoniques. Il découvrait des survivances de préleveurs de dîme saxons et de gendarmes normands à l'ère coloniale. Des origines anglo-saxonnes ${ }^{64}$ étaient attribuées à des phénomènes aussi divers que la Constitution des États-Unis, les formes de gouvernement local et les modèles de colonie agrarienne, à travers le pays.

Comme Frederick Jackson Turner, formé dans ce séminaire, s'en plaindra plus tard, la recherche obsessionnelle des germes teutoniques était «lassante » et le séminaire était devenu « une vraie farce »"5. En 1889 pourtant, alors qu'il était doctorant, il exposa cette théorie dans un compte rendu, affirmant que

[...] le tun de l'ancien allemand était réapparu dans le «village fortifié » du Kentucky et du Tennessee, le folkmoot dans les réunions populaires des colonisateurs, et le witanegemot dans les assemblées représentatives comme la législature de la Transylvanie ${ }^{66}$.

Des plaintes semblables se firent jour par la suite: J. Franklin Jameson, par exemple, confessait qu'il ne possédait pas de

[...] zèle dévorant pour l'étude de l'origine anglo-saxonne du bâton des policiers, du développement historique des permis de colporteurs et d'autres points de l'histoire des institutions ${ }^{67}$,

zèle exigé des participants au séminaire. Un exemple classique montre jusqu'où le séminaire allait dans l'application de la théorie : en 1884, Charles Howard Shinn écrivait une thèse de doctorat sur Mining Camps: A Study in American Frontier Government ; il y poursuit, dans les termes de Herbert Baxter Adams,

[...] la merveilleuse évolution de l'autogouvernement dans les camps miniers en Californie, où les anciennes formes saxonnes de familles associées d'hommes complètement armées retrouvent vigueur et droit ${ }^{68}$. 
Pour Shinn, il faut s'intéresser à la vie sociale des mineurs californiens pour comprendre la "genèse de la croissance de l'État et de la nation" et prendre en compte les «folkmoots de la Sierra». Lorsqu'ils sont étudiés dans cette perspective, les camps miniers de Californie dévoilent "l'impulsion raciale de l'Aryen» et ses «instincts organisateurs héréditaires ", censés être absents des camps non teutoniques ${ }^{69}$. À la fin des années 1880 , la critique interne de la théorie des germes devint fréquente. La critique la plus puissante venait de la plume de Charles Andrews, ancien membre du séminaire. Dans un compte rendu du Domesday Book and Beyond de Maitland, il note qu'une grande leçon de cette œuvre magistrale est que

[...] les institutions anglo-saxonnes n'étaient pas nécessairement porteuses des germes engendrant ce qui est le meilleur et le plus important des idées de l'âge présent, [une leçon qui, pensait-il, sauverait les étudiants] de nombreuses conclusions erronées qui ont trop souvent prévalu ici en Amérique démocratique ${ }^{70}$.

Dès les années 1890 l'école teutonique était, en grande partie, en ruine ${ }^{71}$ et Turner montrait le chemin vers une nouvelle interprétation géo-historique du développement des institutions sociales américaines, dans sa célèbre " thèse de la Frontière ».

Il exista une forte résistance interne à la théorie du germe teutonique, même parmi ceux qui avaient participé, bon gré mal gré, à son élaboration. La question se pose donc de savoir pourquoi elle a pris une telle importance et quelles fonctions elle a remplies pour la profession naissante. Charles Andrews, produit et critique du séminaire, offre quelques indices utiles. Pour Andrews, l'explication de ces croyances est un " présentisme » absolu qui cherche à transférer aux

Allemands primitifs libres et incontrôlés, ces mêmes attributs que les idéaux politiques du XIX ${ }^{e}$ siècle cherchent à rendre réels ${ }^{72}$.

Selon Andrews, les membres du séminaire, poussés par leurs préoccupations idéologiques, projetèrent, sur l'Angleterre médiévale et sur l'Amérique coloniale, une image de communautés fortes, autosuffisantes, individuelles et harmonieuses qu'ils cherchaient à encourager dans l'Amérique de leur propre époque. En ce sens-là, comme le soutient Gettleman,

[...] il devient possible de comprendre des idées comme la théorie du germe, non pas comme des singularités intellectuelles, mais comme les composantes d'une perspective sociale complète, une série plus ou moins cohérente de réponses académiques à la transformation des États-Unis en une puissance industrielle capitaliste, urbanisée, multiraciale et impérialiste ${ }^{73}$.

La théorie du germe teutonique devait fournir un précédent historique, et donc une justification, à un programme entièrement contemporain: assurer un contrôle élitaire des protestants anglo-saxons menacés par une perte d'autorité politique et culturelle ainsi que par un sentiment croissant de faiblesse, face à l'industrialisation et aux populations immigrantes que celle-ci amenait jusqu'aux rives américaines. Le "racisme rhapsodique " implicite et explicite du séminaire de Hopkins, loin d'être accidentel, constituait une espèce de sous-produit de l'érudition historique allemande, importée en premier lieu pour sa supériorité méthodologique et technique. La théorie du germe trouva un foyer accueillant en Amérique, précisément parce qu'elle répondait à des besoins profonds de la part d'une classe professionnelle émergente, alliée aux anciennes élites patriciennes du Nord-Est, dans la défense de son autorité politique et sociale, au moyen d'un renforcement culturel ${ }^{74}$. Elle opère en proposant un modèle de communauté sociale fondé sur le passé, qui ressemble à la notion de Ständeordnung évoquant le Moyen Âge, c'est-à-dire, 
[...] une société hiérarchiquement structurée et fonctionnellement différenciée, mais organiquement unifiée, fondée sur des états sociaux plutôt que sur des individus atomisés et des classes économiques ${ }^{75}$.

En même temps, en tant que forme de «tentative scientifique ", ce modèle permettait à la culture protestante déclinante de faire face au défi du prestige montant de la science du $\mathrm{XIX}^{\mathrm{e}}$ siècle ; avec sa propre science, elle pouvait conserver ses postulats moraux.

Ce mouvement vers une restauration de classe, selon T. J. Jackson Lears, fut renforcé par le débordement sans précédent de racisme que l'on observe parmi les classes moyennes et privilégiées :

En Amérique, comme en Europe, le racisme se combine avec le rejet de la mollesse moderne. Le racisme anglo-saxon offre une explication pour les croisades impérialistes contre des ennemis d'outre-mer « inférieurs » et répond également à des besoins sociaux et psychiques moins évidents. Le racisme réaffirme l'autorité culturelle de la bourgeoisie WASP ; de plus, il fournit peut-être à de nombreux WASP américains une sorte d'identité négative - un moyen de construire une conscience de soi en reniant les impulsions dont ils se méfient. En définissant la paresse, l'irrésolution, l'avarice et d'autres défauts moraux comme des «traits de race " limités à une souche inférieure, les racistes affirment un mode d'identité impérieux et vertueux pour ceux qui ont perdu la conscience solide de soi [...]. De bien des façons, le racisme revigore l'hégémonie de la culture WASP dominante, à un tournant historique critique $^{76}$.

C'est alors que le médiévisme de la profession historique en voie de développement se joignit à l'antimodernisme, dont le porte-parole le plus éloquent fut Henry Adams dans Mont-Saint-Michel and Chartres, une œuvre que F. N. Robinson caractérise comme «l'hommage, sensible et poétique, d'un sceptique, parfois désabusé, à l'esprit du Moyen Âge $\aleph^{77}$. Après 1900, Henry Adams vint au Moyen Âge, garant de l'itinéraire antimoderne du médiévisme. Contrastant nettement avec ses Essays in Anglo-Saxon Law, qui mettent l'accent sur la continuité entre le passé et le présent, Adams cherchait alors, dans le monde médiéval, une vision idéalisée d'un modèle social alternatif, à l'aune de laquelle les défauts du monde moderne pouvaient être jugés ${ }^{78}$. Dans un chapitre célèbre de L'Education, Adams oppose l'esprit de la Vierge, à qui tant de produits artistiques et intellectuels du bas Moyen Âge sont dédiés, à celui de la dynamo moderne, image de la cupidité matérialiste et déshumanisante de la technologie de l'âge moderne ${ }^{79}$. Pour Adams, ce qui est alors attirant dans le Moyen Âge, c'est l'échappatoire qu'il fournit aux réalités de plus en plus rudes du monde moderne, un domaine d'altérité et de fantasme dans lequel on peut retrouver le soi antimoderniste.

La protestation conservatrice convergente, contre les tendances de l'Amérique industrielle et capitaliste de la fin du XIX ${ }^{\mathrm{e}}$ siècle, chez les deux Adams - Henry et Herbert Baxter - montre qu'il existe une relation complexe entre les impulsions modernistes et antimodernistes du médiévisme américain. Ces impulsions trouvent dans le Moyen Âge une contrepartie symbolique aux réflexions sur les implications culturelles de la société moderne, démocratique et industrialisée. Le médiévisme fonctionne ici comme une alternative imaginaire majeure de l'idée américaine de succès matériel laquelle, au lieu de renforcer l'autorité des élites, engendre une richesse nouvelle parmi les parvenus, un conflit de classes d'un type nouveau, une immigration d'étrangers, des troubles dans le monde du travail, et l'idée corrosive que les valeurs spirituelles sont attaquées par la cupidité et la corruption de l'Âge d'or. Donc, l'antimodernisme des médiévistes en Amérique, comme en Grande Bretagne à la même époque, reflète non seulement un point de vue particulier, mais également une classe et une position de pouvoir particulières. 
Issus principalement d'une ancienne souche protestante, ceux qui épousent ses nombreuses variétés sont les leaders moraux et intellectuels de la bourgeoisie WASP américaine qui, comme l'indique Lear,

[...] rejoignent leurs doubles britanniques dans la formation d'une culture victorienne transatlantique [...] pour maintenir les normes et les valeurs dominantes ${ }^{80}$.

À la fois essence des institutions américaines et protestation contre le tour que prend leur évolution, le Moyen Âge est aussi bien précurseur d'un discours modernisant que dépositaire d'une critique antimoderniste. Ainsi se dévoile la durable structure de paradoxe qui marque, au $\mathrm{XIX}^{\mathrm{e}}$ siècle, l'appropriation américaine de l'histoire médiévale. Pendant les premières décennies $d u x^{e}$ siècle, l'itinéraire moderniste du médiévisme triompha et il domina l'étude professionnelle du Moyen Âge, jusqu'aux années soixantedix.

\section{NOTES}

1. «Editor's Preface ", Speculum, 1, 1926, p. 4.

2. Introduction, "Critical Historicism and Medieval Studies» in Lee Patterson (Ed.), Literary Practice and Social Change in Britain, 1380-1530, Berkeley, Los Angeles, Oxford, 1990, p. 2 ; Id., Negotiating the Past: The Historical Understanding of Medieval Literature, Madison, 1987 ; Id., " On the Margin : Postmodernism, Ironic History and Medieval Studies ", Speculum, 65, 1990, p. 87-108.

3. "Critical Historicism and Medieval Studies ", op. cit., p. 2.

4. «Bède's Blush : Postcards from Bali, Bombay, Palo Alto » in John Van Engen (Ed.), The Past and Future of Medieval Studies, Notre-Dame Conferences in Medieval Studies, IV, Notre-Dame, Londres, p. 16.

5. John Van Engen, « An Afterword on Medieval Studies, or the Future of Abelard and Heloise », in John Van Engen (Ed.), op. cit., p. 414.

6. Allen J. Franzten, Desire for Origins: New Language, Old English and Teaching the Tradition, New Brunswick et Londres, 1990, p. 16.

7. Peter W. Williams, "The Varieties of American Medievalism ", Studies in Medievalism, $1, \mathrm{n}^{\circ} 11$, Spring, 1982, p. 8.

8. Bède, A History of the English Church and People, trad. Leo Sherley-Price, rev. par R. E. Latham, New York, 1977, p. 55-56.

9. Allen J. Frantzen, op. cit., p. 16.

10. Jay Fliegelman, Declaring Independence: Jefferson, Natural Language and the Culture of Performance, Stanford, 1993, p. 160-161.

11. Ibid., p. 163.

12. Dorothy Ross, «Historical Consciousness in Nineteenth-Century America ", American Historical Review, 89, 1984, p. 912.

13. Dorothy Ross, The Origins of American Social Science, Cambridge, 1992, p. 24-25.

14. Philip Gleason, "American Catholics and the Mythic Middle Ages ", Keeping the Faith American Catholicism Past and Present, Notre-Dame, Londres, 1987, p. 15.

15. Ibid. 
16. Dorothy Ross, The Origins..., op. cit., p. 24-25; R. W. Southern, The Shape and Substance of Academic History. An Inaugural Lecture Delivered Before the University of Oxford on 2 November, 1961, Oxford, 1961, p. 14.

17. Robin Fleming, "Picturesque History and the Medieval in Nineteenth-Century America ", American Historical Review, 100, 1995, p. 1081.

18. Id, ibid., p. 1076.

19. David Levin, History as Romantic Art: Bancroft, Prescott, Motley and Parkman, Stanford, 1959, p. 74-75.

20. Ibid., p. 76.

21. Ibid., p. 78.

22. Philip Gleason, «American Catholics... », art. cit., p. 12-13.

23. Dorothy Ross, The Origins..., op. cit., p. 54-57.

24. Dorothy Ross, The Origins..., op. cit., p. 58.

25. Robin Fleming, « Picturesque... », art. cit., p. 1077-1078.

26. Ibid., p. 1078.

27. J. C. Levenson, The Mind and Art of Henry Adams, Boston, 1957, p. 18.

28. "The Tendency of History ", The Degradation of the Democratic Dogma, avec une introduction de Brook Adams, New York, 1919, p. 126.

29. William H. Jordy, Henry Adams : Scientific Historian, New Haven, 1952, p. 74.

30. Lettre à Parkman, in J. C. Levenson, op. cit., p. 126.

31. «Darwinism and Modernism : Genetics, Palaeontology, and the Challenge to Progressionism, 1880-1930 ", in Dorothy Ross (Ed.), Modernist Impulses in the Human Sciences 1870-1930, Baltimore et Londres, 1994, p. 236-254. Peter J. Bowler note l'écart entre l'interprétation de l'évolution, datant du retour de la génétique mendélienne vers 1900, et notre interprétation actuelle de Darwin. Darwin lui-même, pense-t-on, n'était pas conscient des implications radicales de sa théorie, c'està-dire qu'il pouvait ne pas exister de but à l'évolution. Il approuva souvent des adaptations lamarckiennes de sa pensée, au gré des situations.

32. The Education of Henry Adams, New York, 1931, op. cit., p. 301.

33. William J. Courtney, "The Virgin and the Dynamo : The Growth of Medieval Studies in North America, 1870-1930», in Francis G. Gentry et Christopher Kleinhenz (Eds), Medieval Studies in North America Past, Present and Future, Medieval Institute Publications, Kalamazoo, 1982, p. 6.

34. Edward Peters a montré comment Georg Waitz dans sa Deutsche Verfassungsgechichte de 1844 a fourni le modèle de la Constitutional History of England de Stubbs. Voir " More Trouble with Henry : The Historiography of Medieval Germany in the Angloliterate World ", Central European History, 28, 1995, p. 52.

35. Herbert Baxter Adams, « New Methods of Study in History ", Journal of Social Science, 18, 1884, p. 244.

36. William H. Jordy, Henry Adams..., op. cit., p. 34-36.

37. Compte rendu de la Constitutional History of England de Stubbs, juillet 1874, in Henry Adams, Sketches for the North American Review, Hamden, Conn., éd. Edward Chalfant, 1986, p. 114.

38. Compte rendu des Historical Essays de Freeman, janv. 1872, in Henry Adams, op. cit., p. 79.

39. J. C. Levenson, The Mind..., op. cit., p. 40.

40. Henry Adams, "The Anglo-Saxon Courts of Law », Essays in Anglo-Saxon Law, Boston, 1876, p. 1.

41. Ibid., p. : 3-5.

42. Adams disait : «L'organisation allemande n'est importante et unique que parce qu'elle n'est pas une organisation tribale mais politique, non pas une tribu, mais un État. » L'idée sous-jacente est explicite, dans son sens à la fois politique et territorial; dans l'usage américain du mot «État»; comme dans "États-Unis », il ne renvoie pas à des limites territoriales, mais à des organisations politiques confédérées ", ibid., p. 3. 
43. Ibid., p. 6.

44. Henry Adams, "The Anglo-Saxon Courts of Law ", op. cit., p. 21.

45. Robin Fleming, "Picturesque... », art. cit., p. 1078.

46. Sur le mélange du germe teutonique et de l'exceptionnalisme américain, voir Dorothy Ross, The Origins of American Social Science, passim, et Adams, " Modemist Social Science in the Land of the New/Old », dans Modernist Impulses in the Human Sciences 1870-1930, op. cit., p. 174-175.

47. Henry Adams, « The Anglo-Saxon Courts of Law », op. cit., p. 45-46.

48. Ibid., p. 37.

49. John Higham, History Professional Scholarship in America, Baltimore et Londres, 1989, (1 $1^{\text {re }}$ éd. 1965), p. 98-99.

50. Pour les chiffres, voir Jurgen Herbst, The German Historical School in American Scholarship, chap. $\mathrm{I}^{\mathrm{er}}$, passim.

51. Ibid., p. 11.

52. Jurgen Herbst, The German Historical School..., op. cit., p. 9.

53. Voir surtout Georg G. Iggers, "The Image of Ranke in American and German Historical Thought ", History and Theory, 2, 1962, p. 17-40.

54. «Leopold von Ranke », Papers of the American Historical Association, III, 1888, p. 104-105.

55. «Is History Past Politics ", Johns Hopkins University Studies in History and Political Science, 13, $\mathrm{n}^{\circ}$ 3-4, 1895, p. 76-77.

56. Pour un récit détaillé de l'enseignement reçu par Baxter Adams en Allemagne, voir Raymond J. Cunningham, "The German Historical World of Herbert Baxter Adams : 1874-1876 ", Journal of American History, 68, 1981, p. 261-275.

57. Jurgen Herbst, The German Historical School..., op. cit., p. 118.

58. John Higham, History Professional Scholarship..., op. cit., p. 94.

59. Ibid., p. 96.

60. Jurgen Herbst, The German Historical School..., op. cit., p. 119.

61. Herbert Baxter Adams, "The Germanic Origin of New England Towns ", Johns Hopkins University Studies in Historical and Political Science, 1, 1883, p. 8.

62. Marvin Gettleman (Ed.), The Johns Hopkins University Seminary of History and Politics. The Records of an American Educational Institution 1877-1912, 5 vol., New York et Londres, 1987, p. 17-18.

63. Ibid., p. 16.

64. Baxter Adams affirmait que « les réunions des fermiers teutoniques dans les champs, en vue de la distribution de terre et de la régulation des moissons (allusion à Tacite) étaient les germes des réunions paroissiales anglaises et des assemblées de villes en Nouvelle-Angleterre... ", "The Germanic Origin of New England Towns », op. cit., p. 18.

65. Lettre à son père, citée par Jurgen Herbst, The German Historical School..., op. cit., p. 107.

66. Robert E. Lemer, "Tumer and the Revolt Against E. A. Freeman ", Arizona and the West, 5, 1963, p. 104.

67. Marvin Gettleman (Ed.), The Johns Hopkins University Seminary..., op. cit., p. 24.

68. Herbert B. Adams, "Cooperation in University Work ", Johns Hopkins University Studies..., op. cit., 1, 1883, p. 44-45 ; Marvin Gettleman (Ed.), The Johns Hopkins University Seminary..., op. cit., p. : 22-23.

69. Ibid., p. 23.

70. Robin Fleming, «Picturesque...», art. cit., p.1091; Dorothy Ross, « Historical Consciousness... », art. cit., p. 927.

71. John Higham, History Professional Scholarship..., op. cit., p. 161.

72. Marvin Gettleman (Ed.), The Johns Hopkins University Seminary..., op. cit., p. 32-33.

73. Marvin Gettleman (Ed.), The Johns Hopkins University Seminary..., op. cit., p. 34.

74. Dorothy Ross, "On the Misunderstanding of Ranke and the Origins of the Historical Profession in America », p. 35. 
75. Philip Gleason, « American Catholics... », op. cit., p. 32.

76. T. J. Jackson Lears, No Place of Grace Antimodernism and the Transformation of American Culture 1890-1920, Chicago et Londres, 1994 ; $1^{\text {re }}$ éd. 1983, p. 108.

77. F. N. Robinson, "Anniversary Reflections ", Presidential Address delivered at the TwentyFifth Annual Meeting of the Medieval Academy, Speculum, 25, 1950, p. 494.

78. Philip Gleason, «American Catholics... », op. cit., p. 20. Le manque de place ne me permet pas de suivre le développement du médiévisme catholique aux États-Unis, avant le néo-thamisme, qui s'est développé largement hors des tendances dominantes de l'université : voir l'article de Gleason déjà cité, voir aussi John Van Engen, « The Christian Middle Ages as an Historiographical Problem », American Historical Review, 91, 1986, p. 519-552.

79. William J. Courtney, «The Virgin and the Dynamo : The Growth of Medieval Studies in North America 1870-1930 », Francis G. Gentry et Christopher Kleinhenz (Eds), Medieval Studies in North America Past Present, and Future, Kalamazoo, MI, 1982, p. 21.

80. T. J. Jackson Lears, No Place of Grace, op. cit., p. XVI. 